\title{
Thyroid fine-needle aspiration: the relevance of BRAF mutation testing
}

\author{
Giuseppe Di Benedetto
}

Received: 15 January 2014/ Accepted: 18 February 2014/Published online: 4 March 2014

(C) Springer Science+Business Media New York 2014

The manuscript "Additional BRAF mutation analysis may add additional diagnostic value in thyroid nodules with suspicion of malignant cytology alone even when the nodules do not show suspicious US features" [1] pointed out that some nodules, which were not considered suspicious on the ultrasound scan, can be potentially malignant.

Moreover, the authors performed the V600E-BRAF test on the samples which were diagnosed in cytology as at variance with undetermined significance/follicular lesion (AUS/FLUS), to prove it.

This study proves that nodules which are not suspicious on the ultrasound scan cannot reassure the clinician, altogether. Therefore, it is always necessary to check carefully all the thyroid nodules. The V600E-BRAF tests advantage is that it helps the cytopathologist to diagnose the papillary carcinoma and the follicular variant. The V600-Braf mutation is considered a specific marker of the thyroid papillary carcinoma. Concerning the role of mutation on the management of thyroid nodules, many studies have been published with no definitive conclusion.

Therefore, it would be valuable to research this topic in more detail so as to clearly define the role of the V600 Braf test when performed on Fine-needle cytology.

G. Di Benedetto $(\bowtie)$

Cytopathology Service, Department of Clinical Pathology,

Clinical Hospital Aversa (Caserta), Caserta, Italy

e-mail: diben.g@libero.it

\section{What is the role of V600E-BRAF mutation on Thyroid FNC?}

On the one hand, several studies [2] have proven that the V600E-BRAF mutation is closely related to high-risk clinico pathological factors and poorer outcome of PTC, and this suggests that this mutation should be considered as a poor prognostic marker in PTC, which may lead to better management of individual patients.

On the other hand, several studies have demonstrated that BRAF mutation testing of thyroid fine-needle aspiration specimens enhances the predictability of malignancy in thyroid follicular lesions of indeterminate significance, which are known to have an excellent prognosis [3-6].

Some authors $[7,8]$ in their publications have underlined this contradiction.

It is important that a resolution of this contradiction be determined.

The overall outcome on patients with papillary carcinoma is excellent. The V600 BRAF mutation is positive in nearly $70 \%$ papillary carcinoma, therefore showing that most PTCs, with BRAF mutation are not aggressive. It is clearly wrong to suggest that this mutation should be considered as a poor prognostic marker in PTC. That BRAF mutant PTCs also include those that are aggressive does not mean that the PTCs with the BRAF mutation have a poor prognosis.

In conclusion, there is a misconception among pathologists and clinicians, who believe that BRAF positive means aggressive. It does not. If there was a correlation between prognosis and BRAF mutation, considering that nearly $70 \%$ of papillary carcinomas have V600-BRAF mutation, we would have the $70 / 80 \%$ of papillary carcinomas with poor prognosis, but we do not. 
Is there a correlation between suspicious ultrasound features V600E-BRAF mutation?

A misconception arose with the publication [3] "Outcome of Thyroid Nodules Characterized as Atypical of Undetermined Significance or Follicular Lesion of Undetermined Significance and Correlation With Ultrasound Features and V600E-BRAF Mutation Analysis".

The authors assert that the nodules with suspicious features on ultrasound have a higher malignancy rate than those with indeterminate features on ultrasound, using the V600-BRAF test to prove it.

This statement does not hold water, and I think, as a pathologist, that it points to an obsession of experts of image analysis to arrive at certain diagnoses just from the processing of X-ray and ultrasound scans. This attitude has already produced many problems related to the diagnostics.

However, considering that the nodules with suspicious ultrasound features have a higher malignity predictive value, it's obvious to observe more V600 BRAF mutated with non-suspicious nodules.

A publication [9] titled "BRAF mutation analysis and sonography as adjuncts to fine-needle aspiration cytology of papillary thyroid carcinoma: their relationships and roles" gives an answer to the question, concluding that the sonography features of PTC are not related to BRAF status and that the combination of sonography and BRAF testing would increase the diagnostic accuracy of cytological diagnoses of PTC.

\section{In the literature, the V600 BRAF mutation papillary carcinomas of thyroid were detected between 30 and 75 percent, why does this happen?}

To achieve an optimal result for use in clinical practice [10], aspirations of the thyroid are best performed with 25 or 23 gauge needles by an experienced practitioner. It would be better that fine-needle cytology was carried out under ultrasound guidance aided by an echographist and cytopathologist. The issue of what constitutes an adequate thyroid sample has been a subject of debate for several years. Six clusters of epithelial cells on one smear constitute an acceptable minimum of sampling adequacy. The residual material is put in a test tube [10].

The amount of DNA, extracted from the test tube, would be greater than $80 \mathrm{ng}$ for every specimen. For the evaluation of the validity of DNA samples, we suggest an amplification of a region of the thyreoglobulin gene. The presence of the gene proves the adequacy of the sample.

Following this protocol, the mutation would be found in most of the papillary carcinomas.
I believe that the reason why authors have not detected most V600-BRAF mutation in papillary carcinomas diagnosed is due to an incorrect sampling procedure. The DNA extraction must be performed by a single fine-needle cytology sample [11].

\section{Is it useful to perform the FNC and V600-Braf test on nodules without suspicious ultrasound features?}

Yes it is because there are several malignant nodules without suspicious ultrasound features.

So I believe it is really important that this study [1] has been published in this journal because it would open an interesting debate on the evaluation of the cost/benefit ratio.

In this perspective, according to my advice, the V600BRAF test should be performed only on the undetermined nodules to find the follicular mutations of the papillary carcinoma.

\section{References}

1. J.Y. Seo, E.-K. Kim, J.Y. Kwak, Additional BRAF mutation analysis may have additional diagnostic value in thyroid nodules with "suspicious for malignant" cytology alone even when the nodules do not show suspicious US features. Endocrine (2014). doi:10.1007/s12020-013-0150-5

2. R. Elisei, D. Viola, L. Torregrossa, R. Giannini, C. Romei, C. Ugolini, E. Molinaro, L. Agate, A. Biagini, C. Lupi, L. Valerio, G. Materazzi, P. Miccoli, P. Piaggi, A. Pinchera, P. Vitti, F. Basolo, The BRAF(V600E) mutation is an independent, poor prognostic factor for the outcome of patients with low-risk intrathyroid papillary thyroid carcinoma: single-institution results from a large cohort study. J. Clin. Endocrinol. Metab. 97, 4390-4398 (2012)

3. S.H. Jeong, H.S. Hong, E.H. Lee, J.G. Cha, J.S. Park, J.J. Kwak, Outcome of Thyroid Nodules Characterized as Atypia of Undetermined Significance or Follicular Lesion of Undetermined Significance and Correlation With Ultrasound Features and BRAF(V600E) Mutation Analysis. AJR Am. J. Roentgenol. 201, 854-860 (2013)

4. D. Barbaro, R.M. Incensati, G. Materazzi, G. Boni, M. Grosso, E. Panicucci, P. Lapi, C. Pasquini, P. Miccoli, The BRAF V600E mutation in papillary thyroid cancer with positive or suspected pre-surgical cytological finding is not associated with advanced stages or worse prognosis. Endocrine (2013). doi:10.1007/ s12020-013-0029-5

5. T.H. Kim, Y.J. Park, J.A. Lim, H.Y. Ahn, E.K. Lee, Y.J. Lee, K.W. Kim, S.K. Hahn, Y.K. Youn, K.H. Kim, B.Y. Cho, J. Park do, The association of the BRAF(V600E) mutation with prognostic factors and poor clinical outcome in papillary thyroid cancer: a meta-analysis. Cancer 118(7), 1764-1773 (2012)

6. G. Di Benedetto, A. Fabozzi, C. Rinaldi, Clinical management of thyroid nodules with indeterminate cytology: our institutional experience using SIAPEC cytological criteria and V600-BRAF test. Pathologica 105(1), 1-4 (2013) 
7. J.K. Nam, C.K. Jung, B.J. Song, D.J. Lim, B.J. Chae, N.S. Lee, W.C. Park, J.S. Kim, S.S. Jung, J.S. Bae, Is the BRAF(V600E) mutation useful as a predictor of preoperative risk in papillary thyroid cancer? Am. J. Surg. 203(4), 436-441 (2012)

8. J.P. Lopes, E. Fonseca, BRAF gene mutation in the natural history of papillary thyroid carcinoma:diagnostic and prognostic implications. Acta. Med. Port. 24(Suppl 4), 855-868 (2011)

9. W.J. Moon, N. Choi, J.W. Choi, S.K. Kim, T.S. Hwang, BRAF mutation analysis and sonography as adjuncts to fine-needle aspiration cytology of papillary thyroid carcinoma: their relationships and roles. AJR Am. J. Roentgenol. 198(3), 668-674 (2012)

10. E. Puxeddu, S. Filetti, BRAF mutation assessment in papillary thyroid cancer: are we ready to use it in clinical practice? Endocrine (2013). doi:10.1007/s12020-013-0139-0

11. G. Di Benedetto, A. Fabozzi, C. Rinaldi, C.R. Rinaldi, BRAF test and cytological diagnosis with a single fine needle cytology sample. Acta. Cytol. 57(4), 337-340 (2013) 